\title{
Theory of the thermally-stimulated-current transport peak. Application to a dispersive transport case
}

\author{
J. Plans,* M. Zieliński, and M. Kryszewski \\ Polish Academy of Sciences, Centre of Molecular and Macromolecular Studies, 90-362 Kódź, Boczna 5, Poland
}

(Received 7 August 1980)

\begin{abstract}
Thermally-stimulated-current (TSC) technique consists of heating a dielectric, with excess carriers having been introduced. The current flowing under the effect of applied electric field is then recorded as a function of increasing temperature. The current maximum obtained is called a "transport TSC peak" if it appears due to thermally activated transport of carriers initially generated at one electrode and collected at the other. In this paper the transport of carriers, controlled by multiple trapping, is studied when the temperature of the system is increased linearly with time. The general equations for current density in a TSC experiment are obtained and are applied for the cases of a single trap and an exponential trap distribution. The condition for a TSC current maximum, initial rise of current, and partial heating technique are studied in detail. The properties of the transport TSC peak differ considerably from those of the classical TSC; the position of the current maximum and activation energy found from partial heating at the maximum depend on the field applied and on sample thickness: It is also found that there exists a correlation between the transport TSC peak and time-of-flight signal, and the transport parameters found from TSC (activation energy of mobility, parameter of dispersion $\alpha$, etc.) correspond to those of the time of flight measurements. The critical trap criterion and concepts of lifetime and transit time are extended to the general case of nonisothermal transport.
\end{abstract}

\section{INTRODUCTION}

In order to introduce the concept of a thermallystimulated-current (TSC) transport peak we recall the TSC procedure in the case of phenomena involving only charge carriers (excluding dipolar motion). The sample of semiconductor or dielectric, maintained at a certain (low) temperature $T_{0}$, is subjected to excitation, and charge carriers, in excess of the thermal equilibrium ones, are introduced into the system under investigation. The excess carriers are immobilized at $T_{0}$ in some traps. During the temperature rise two competitive processes become operative: release of carriers from traps, causing increase of the mobile carrier density, unless the source of carriers is exhausted, and another process decreasing the number of carriers available for transport. These two processes are accordingly responsible for the rise and subsequent fall of the current, causing the appearance of a TSC peak.

Despite various excitation sources (electrode injection, photogeneration, photoinjection, corona charging, etc.) this description of a TSC peak is fairly general, and most of the TSC experiments described in the literature fit this scheme. The main difference between various experimental modifications of this technique, and hence in the interpretation of results, requires an understanding of the second process causing the drop of current. The classical approach ${ }^{1-3}$ assumes for this process deep trapping, fast transport in the conduction band, or recombination in the bulk, which allows us to obtain information on the depths, densities, and eventually the distribution of traps, releasing the charge carriers.

Another more recent approach ${ }^{4-6}$ assumes that carriers are transported through the sample unless they reach the electrode, and the decrease of the TSC current is due to absorption of the carriers by the electrode. This approach allows us to derive information on the transport process which is now of considerable interest.

In this paper the last concept is developed in some more detail. We assume that we deal with one type of carriers initially generated with a surface density $\sigma$ at the electrode. Upon heating they are transported under external field unless they reach the collecting electrode. Thus the experimental conditions are similar to those of "time-of-flight" experiments, except for the temperature variation in the case of TSC measurements. This allows us to make use of the theory of transient photoconductivity, on which. subject marked progress has been made recently, ${ }^{7-10}$ and to develop a theory of the TSC peak.

The general theory, which will be presented in Sec. II, is then applied to the case of dispersive transport, which is known to produce, in the time-of-flight experiments, current traces of the type:

$$
i(t) \sim \begin{cases}t^{-(1-\alpha)}, & t<t_{\tau} \\ t^{-(1+\alpha)}, & t>t_{\tau}\end{cases}
$$

where $t_{\tau}$ is the experimental transit time and $\alpha$ is the experimental dispersion parameter. It was shown ${ }^{7}$ that for this kind of a trace the motion of 
the sheet of carriers, initially produced at the electrode by a pulse of light, cannot be described by conventional Gaussian statistics. It was further found that the broad dispersion of current traces can be well explained by the band model, with traps provided so that the carrier traveling through the sample is captured approximately once in a trap whose mean release time is around the experimental transit time $t_{\tau}{ }^{8}$ This statement is called the critical-trap criterion (CTC).

In this paper the concept of the critical trap is used in developing the theory of a TSC transport peak, and it will be shown that the critical-trap criterion also holds for distinguishing a dispersive transport peak from a nondispersive one. The concepts of release temperature and transit temperature will be introduced, corresponding to lifetime and transit time in an isothermal experiment, in order to facilitate understanding of the processes leading to appearance of the transport TSC peak.

It will be shown that properties of such a peak differ considerably from that predicted by the classical theories, allowing us easily to verify the origin of the experimental maxima and thus to assign to certain internal variables of the investigated system the parameters obtained.

\section{THEORY}

\section{A. General remarks}

A theory of the TSC transport peak, based on the concepts and solutions derived for isothermal transport, ${ }^{8-10}$ is developed. Although the formalism used hereafter is associated with the bandtransport scheme, it can be easily extended to accommodate the hopping-transport concept as well, if the term trap is used for any localized state which immobilizes a charge carrier for a considerable length of time, while the transport state is any state which determines the microscopic mobility of a charged carrier. In other words, we can say that traps are states which are sufficiently distant in space to prevent any direct transitions between them, and transport states are, on the contrary, close enough to each other to maintain a continuous drift velocity for a carrier. ${ }^{8}$

A plane-parallel sample is assumed (having thickness $L$ ) in which at time $t=0$ a sheet of carriers with a surface density $\sigma$ is generated at one of the contacts. The temperature is then increased linearly with time,

$$
T=T_{0}+\beta t,
$$

where $\beta$ is the heating rate. Only one type of carrier is taken into consideration, allowing us to neglect recombination, and space-charge effects are not considered assuming the small-signal case. The carriers at temperature $T_{0}$ are immediately trapped near the electrode and begin to move upon the increase of temperature.

In the simplest models of TSC, trap levels well separated in energy are assumed. It allows us to obtain a simple solution for TSC current considering a particular level which supplies carriers under actual conditions. The shallower traps are in equilibrium with transport states and the deeper ones are acting only as centers trapping the carriers definitively, with a lifetime greater than the time of experiment. The case of traps continuously distributed in the energy gap is more complicated. It is necessary to consider the trapping and detrapping processes into, and from, all the levels. However, one can generalize the former approach for a continuous distribution also and divide the distributed traps into two regions:

(i) The shallow trap region between the conduction-band edge and the temperature-dependent release energy $\bar{\epsilon}(T)$; in this region thermal equilibrium can be assumed.

(ii) The region of deep traps having depth greater than $\bar{\epsilon}(T)$ in which the trapped carriers do not have enough time to escape in the time of experiment at temperature $T$.

The release energy $\bar{\epsilon}(T)$ is the depth of this trap for which the escaping rate has its maximum at temperature $T$. For constant temperature the release energy $\bar{\epsilon}(t)$ is defined as ${ }^{10}$

$$
[\nu(\bar{\epsilon}) \exp (-\bar{\epsilon} / k T)]^{-1}=t,
$$

where $\nu(\bar{\epsilon})$ is the attempt-to-escape frequency (eventually activation energy dependent) and $t$ is the time counted from the moment of carrier injection. The left-hand term denotes the lifetime for the traps with activation energy. $\bar{\epsilon}$. Under a constant heating-rate condition the release energy $\bar{\epsilon}(T)$ is defined as follows:

$$
\frac{1}{\beta} \int_{T_{0}}^{T} \nu(\bar{\epsilon}) \exp (-\bar{\epsilon} / k T) d T=1,
$$

which reduces to Eq. (3) for constant temperature $(\beta=0)$. This last expression allows us to introduce another concept of the release temperature which will be very useful in further analysis. The release temperature $T_{r}$ of a particular trap is the temperature at which the rate of escaping carriers from this trap reaches its maximum. In this case $T$ in Eq. (4) denotes release temperature and $\bar{\epsilon}$ is the trap depth. The definition of release temperature reduces to that of lifetime for $\beta=0$. Thus for traps above $\bar{\epsilon}(T)$ thermal 
equilibrium can be assumed because of the large number of capture or release events at temperature $T$. For traps deeper than $\bar{\epsilon}(T)$ it is unlikely that the carriers trapped in them will escape until the corresponding release temperatures are reached.

\section{B. The equations of the TSC transport peak}

The equations describing the motion of one type of carriers through transport states and controlled by capture in traps distributed in energy are

$$
\begin{aligned}
& \beta \frac{\partial p(x, T)}{\partial T}+\mu E \frac{\partial p_{0}(x, T)}{\partial x}=0, \\
& \beta \frac{\partial \rho(x, T, \epsilon)}{\partial T}=\omega(\epsilon) p_{0}(x, T)-r(\epsilon, T) \rho(x, T, \epsilon),(6) \\
& p(x, T)=p_{0}(x, T)+\int_{0}^{\infty} \rho(x, T, \epsilon) d \epsilon,
\end{aligned}
$$

where $x$ is the coordinate in the drift direction with electrodes at $x=0$ and $x=L, p_{0}(x, T)$ is the local population of free carriers in transport states, $p(x, T)$ is the total population of carriers, $\rho(x, T, \epsilon)$ is the population of carriers trapped in traps having activation energy $\epsilon$ and $\mu$ is the microscopic mobility. The continuum of traps is characterized by the trap parameters $\omega(\epsilon)$ and $r(\epsilon, T)$ defined as follows:

$$
\begin{aligned}
& \omega(\epsilon)=n(\epsilon) \sigma_{c s}(\epsilon) v_{a}=C(\epsilon) m(\epsilon), \\
& r(\epsilon, T)=\nu(\epsilon) \exp (-\epsilon / k T),
\end{aligned}
$$

where $n(\epsilon)$ is the density of traps with activation energy $\epsilon, \sigma_{c s}(\epsilon)$ is their capture cross section, $v_{a}$ is the average velocity of the mobile carrier, and $\epsilon$ is the activation energy of the trap under consideration. The parameter $C$ characterizes the probability of capture. The trap parameters are defined so that for $T=$ const $[r(\epsilon)]^{-1}$ represents the mean residence time, in traps of activation energy $\epsilon$, and [0 $\left.\int^{\infty} \omega(\epsilon) d \epsilon\right]^{-1}$ is the mean travel time between trapping events. One should also note that $\omega(\epsilon)$ and $n(\epsilon)$ are distributions in activation energies. When the effect of the electric field is due to barrier modification (as in the Poole-Frenkel case) a relation between $\nu(\epsilon)$ and $C(\epsilon)$ can be used provided by detailed balancing, ${ }^{8}$

$$
C(\epsilon)=\nu(\epsilon) / N_{0},
$$

where $N_{0}$ is the concentration of transport states. It can be shown that this assumption is not essential for the theory, which can be developed also without the use of Eq. (10). Equation (6) can now be rewritten as

$\beta \frac{\partial \rho(x, T, \epsilon)}{\partial T}=C(\epsilon)\left[n(\epsilon) p_{0}(x, T)-N_{0} e^{-\epsilon / k T} \rho(x, T, \epsilon)\right]$.
As was assumed, the charges are generated with a surface density $\sigma$ at $x=0$ and are collected by the other electrode at $x=L$. Once generated at the electrode at low initial temperature, the carriers escape from the surface and are trapped close to the electrode. The boundary and initial conditions for TSC are then

$$
\begin{aligned}
& p_{0}(0, T)=\frac{\sigma \beta}{\mu E} \delta\left(T-T_{0}\right), \\
& p_{0}\left(x, T_{0}\right)=0 \\
& \rho\left(x, T_{0}, \epsilon\right)=0
\end{aligned}
$$

In order to develop the TSC equations we use now the concept of release energy $\bar{\epsilon}(T)$. Thermal equilibrium for levels $\epsilon<\bar{\epsilon}(T)$ implies

$$
\frac{\partial \rho(x, T, \epsilon)}{\partial T} \simeq 0,
$$

and from (11) we get

$\rho(x, T, \epsilon)=\frac{1}{N_{0}} n(\epsilon) \exp (\epsilon / k T) p_{0}(x, T), \epsilon<\bar{\epsilon}(T)$.

For traps below the release energy $\epsilon>\bar{\epsilon}(T)$ we can neglect in (11) the release term and take into consideration only the capture process, which yieids

$\rho(x, T, \epsilon)=\frac{C(\epsilon) n(\epsilon)}{\beta} \int_{T_{0}}^{T} p_{0}\left(x, T^{\prime}\right) d T^{\prime}, \quad \epsilon>\bar{\epsilon}(T)$.

Putting (15) and (16) into (7) we obtain after rearrangements

$$
p(x, T)=\mu E\left(\frac{p_{0}(x, T)}{v(T)}+\frac{\lambda(T)}{v(T)} P_{0}(x, T)\right),
$$

where $P_{0}(x, T), \lambda(T)$, and $v(T)$ are defined for convenience as

$$
\begin{aligned}
& P_{0}(x, T)=\frac{1}{\beta} \int_{T_{0}}^{T} p_{0}\left(x, T^{\prime}\right) d T^{\prime}, \\
& v(T)=\mu E\left[1+\frac{1}{N_{0}} \int_{0}^{\bar{\epsilon}(T)} n(\epsilon) \exp \left(\frac{\epsilon}{k T}\right) d \epsilon\right]^{-1}, \\
& \lambda(T)=\frac{v(T)}{\mu E} \int_{\bar{\epsilon}(T)}^{\infty} C(\epsilon) n(\epsilon) d \epsilon .
\end{aligned}
$$

One should observe that $v(T)$ represents the drift velocity of a carrier in thermal equilibrium between transport states and traps having depth $\epsilon$ smaller than $\bar{\epsilon}(T)$. Using (18) we can get from (5):

$$
\beta \frac{\partial p(x, T)}{\partial T}=-\beta \mu E \frac{\partial^{2}}{\partial x \partial T} P_{0}(x, T),
$$

which with the initial conditions (13) yields

$$
p(x, T)=-\mu E \frac{\partial P_{0}(x, T)}{\partial x}
$$

Substituting $P_{0}(x, T)$ taken from (17) into (21) and using (5) we obtain an equation for the total 
carrier density $p(x, T)$ :

$$
\beta \frac{\partial p(x, T)}{\partial T}+v(T) \frac{\partial p(x, T)}{\partial x}+\lambda(T) p(x, T)=0,
$$

with the initial condition (13) and the boundary condition

$$
p(0, T)=\sigma \frac{\lambda(T)}{v(T)}+\sigma \frac{\beta \delta\left(T-T_{0}\right)}{v(T)},
$$

which results from (12), (17), and (18). Integrating $\mathrm{Eq}$. (23) in $x$ and using (24) we obtain

$$
\int_{0}^{\infty} p(x, T) d x=\sigma
$$

as should be expected, for the total charge is conserved.

The mean position of the packet is defined as

$$
\bar{x}(T)=\frac{\int_{0}^{\infty} x p(x, T) d x}{\int_{0}^{\infty} p(x, T) d x} .
$$

Integrating Eq. (23) after multipling by $x$ and using (24), (25), and (26) we get an equation for the evolution in space of the mean of the packet

$$
\beta \frac{d \bar{x}(T)}{d T}+\lambda(T) \bar{x}(T)=v(T)
$$

with the initial condition $\bar{x}\left(T_{0}\right)=0$, which results from (12) and (13). The solution of this equation yields the low motion of the mean

$$
\bar{x}(T)=\frac{1}{\beta} \frac{\int_{T_{0}}^{T} v\left(T^{\prime}\right) \exp \left[1 / \beta \int_{T_{0}}^{T^{\prime}} \lambda\left(T^{\prime \prime}\right) d T^{\prime \prime}\right] d T^{\prime}}{\exp \left[1 / \beta \int_{T_{0}}^{T} \lambda\left(T^{\prime}\right) d T^{\prime}\right]} .
$$

The experimentally measured quantity is the total current density

$$
j(T)=\frac{e \mu E}{L} \int_{0}^{L} p_{0}(x, T) d x
$$

which, with the use of (5) and (12), can be rewritten as

$$
j(T)=\frac{-e \beta}{L} \frac{\partial}{\partial T} \int_{0}^{L}(L-x) p(x, T) d x .
$$

It can be observed that for dispersive transport the velocity of the mean $\beta d \bar{x} / d T$ is usually much smaller than the drift velocity $v(T)$ of a carrier. In this case Eq. (28) describing the position of the mean takes a simple form

$$
\bar{x}(T)=v(T) / \lambda(T),
$$

and an approximate solution describing the carrier packet can be obtained

$$
\begin{aligned}
& p(x, T)=\sigma \frac{\lambda(T)}{v(T)}\left(1-\frac{x}{\frac{1}{\beta} \int_{T_{0}}^{T} v\left(T^{\prime}\right) d T^{\prime}}\right)^{\left(\frac{\lambda(T)}{v(T)} \frac{1}{\beta} \int_{T_{0}}^{T} v\left(T^{\prime}\right) d T^{\prime}\right)^{-1}} \text { for } 0 \leqslant x \leqslant \frac{1}{\beta} \int_{T_{0}}^{T} v\left(T^{\prime}\right) d T^{\prime}, \\
& p(x, T)=0 \text { for } \frac{1}{\beta} \int_{T_{0}}^{T} v\left(T^{\prime}\right) d T^{\prime} \leqslant x<\infty,
\end{aligned}
$$

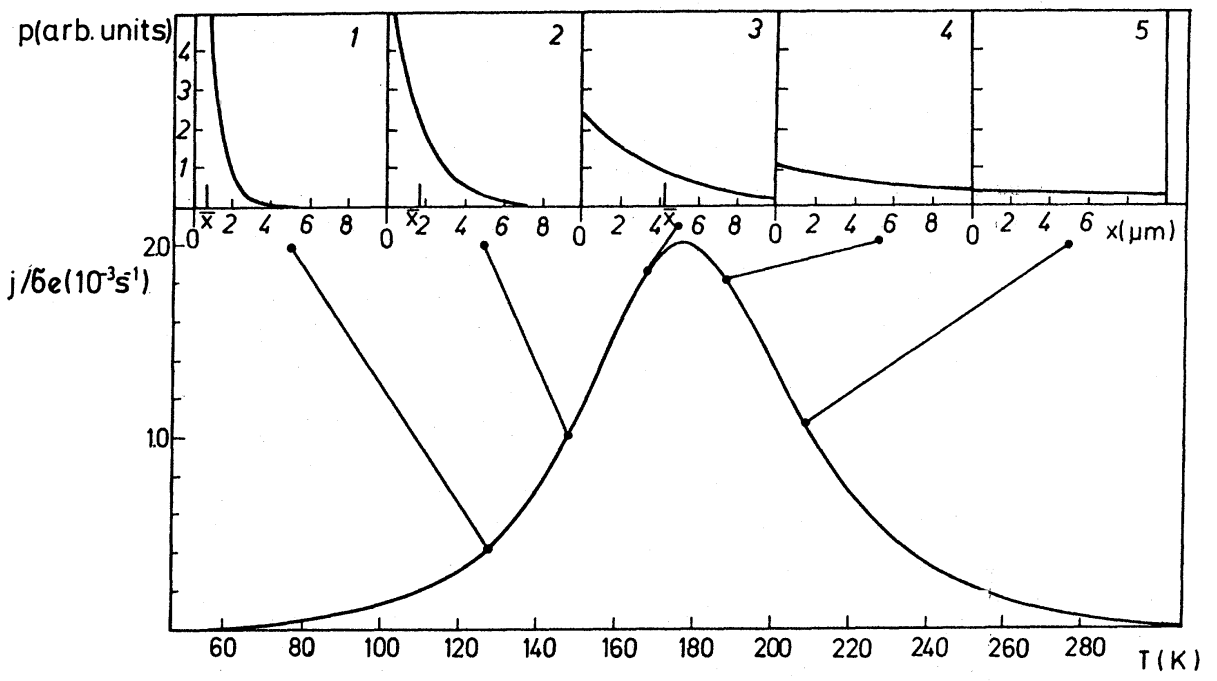

FIG. 1. Transport TSC peak and the profiles of the carrier packet at several temperatures, indicated at the plot. Parameters used for calculation: $\mu=10^{-6} \mathrm{~m}^{2} / \mathrm{V} \mathrm{s}, N_{0}=10^{25} \mathrm{~m}^{-3}, C_{0}=5 \times 10^{-12} \mathrm{~m}^{3} / \mathrm{s}, \epsilon_{0}=0.07 \mathrm{eV}, \beta=0.15 \mathrm{~K} / \mathrm{s}$. 
which fulfills the boundary (24) and initial (13) conditions and implies the conservation of the total charge [Eq. (25)]. It also yields the expected mean position for the packet [Eq. (31)]. It can be seen that the packet becomes zero for distances farther than that which is reached by the fastest carrier $(1 / \beta) \int_{T_{0}}^{T} v\left(T^{\prime}\right) d T^{\prime}$. The TSC current shape and corresponding carrier packets calculated from Eqs. (30) and (32) for dispersive transport are presented in Fig. 1.

When the carriers have not yet reached the collecting electrode, Eq. (30) reduces to

$$
j(T)=\frac{e \sigma \beta}{L} \frac{d \bar{x}(T)}{d T},
$$

which yields the initial rise current.

This completes the set of general equations [Eqs. (23)-(33)] describing the TSC transport peak. In the following section the properties of the peak (maximum condition, initial rise, etc.) will be studied in detail.

\section{The TSC maximum condition}

Upon the increase of temperature the carrier packet begins to advance toward the collecting electrode and the current rises, for the motion of $\bar{x}$ is accelerated. Initially, all the carriers are inside the sample. Since the fastest carrier reaches the electrode, an increasing number of carriers is absorbed. A moment arrives when increase of the carrier density caused by emptying the traps is compensated for by the loss of carriers absorbed by the electrode, yielding the current maximum. For the isothermal case it was shown ${ }^{10}$ that the break in the time-of-flight signal corresponding to the transit time appears for exponential distribution of traps when the mean of the carrier packet reaches the electrode, i.e., for $x \simeq L$ (exactly for $x=L / \sqrt{2}$ ). On the other hand, by means of numerical computation, it was shown ${ }^{11}$ that by transforming the time-of-flight signal into a TSC plot, the transit time corresponds to current maximum. Thus we can say that the TSC maximum appears at the temperature at which the mean of the packet reaches the collecting electrode,

$$
\bar{x}\left(T_{m}\right) \simeq L,
$$

and, by analogy to time of flight, the temperature $T_{m}$ will be called the transit temperature. This relation is approximate, and numerical calculations using Eqs. (30) and (32) show also that in the case of the TSC peak the distance traveled by the mean $\bar{x}$ at the TSC maximum is a thickness reduced by small factor, ca. $\sqrt{2}$, which confirms the correspondence of time of flight and transport TSC. Since in real experiments carriers are usually distributed in space, we define an effective thickness which in the ideal case is equal to $L_{\text {expt }} / \sqrt{2}$ and in the experimental case is reduced by a factor characterizing the initial displacement of the mean. In further discussion $L$ will denote the effective thickness.

In order to find the position of the mean at any temperature one should use Eq. (28), which can be easily solved numerically. However, provided Eq. (31) can be used (dispersive transport) one immediately obtains a simple maximum condition

$$
L=v\left(T_{m}\right) / \lambda\left(T_{m}\right),
$$

which with the use of (19) and (20) can be rewritten as

$$
L=\frac{\mu E}{\int_{\bar{\epsilon}\left(T_{m}\right)}^{\infty} C(\epsilon) n(\epsilon) d \epsilon} .
$$

The physical meaning of this last expression is that the maximum temperature is reached when the mean travel distance of a carrier, before it is retrapped in a trap having a depth larger than the release energy at this temperature $\bar{\epsilon}\left(T_{m}\right)$, is equal to the effective thickness. Until $T_{m}$ is reached, the carriers are consecutively released from traps of depth $\bar{\epsilon}(T)$ and retrapped in traps of depth $\epsilon>\bar{\epsilon}(T)$ before being able to arrive at the collecting electrode. The trapping parameter $\omega(\epsilon)$ is a decreasing function of trap depth; or, in other words, the deeper the trap, the longer the mean travel time $\omega^{-1}(\epsilon)$ between trapping events. The temperature $T_{m}$ is reached at a moment at which the carriers released from a trap of depth $\bar{\epsilon}\left(T_{m}\right)$ do not have enough time to be trapped in a trap of the same or greater depth, unless they travel the distance equal to thickness $L$. The trap having depth $\bar{\epsilon}\left(T_{m}\right)$ is called a critical trap $\epsilon_{c}$ and is defined in analogy to the isothermal case ${ }^{8}$ as a trap which captures a carrier approximately once before it travels through the sample to the collecting electrode. The release temperature of this critical trap is the transit temperature and it is equal to the maximum temperature of a transport TSC peak.

In the case of a discrete distribution with several levels of traps well separated in energy, it is not reasonable to expect a single maximum in TSC spectrum. Nevertheless, the same concepts and assumptions used for developing the former theory can now easily be applied to the TSC transport process involving a discrete distribution of traps. About the $i$ th release temperature, corresponding to the set of $i$ traps, a maximum will appear, or rather a shoulder if the several release temperatures are not enough separated. The carriers will be consecutively liberated from the 
trapping levels at their respective release temperatures and retrapped into deeper traps with a tendency to occupy a set of traps with a greater probability of capture $C_{i}$ and concentration $N_{i}$, i.e., with greater trap parameter $\omega_{i}=C_{i} N_{i}$. Between all the maxima (or shoulder) temperatures which appear in the TSC spectrum there is one corresponding to the exit of the packet out of the sample. This maximum is described by condition (34) and, because of this, it deserves the right to be called the "transit temperature." This transit temperature is controlled by releasing carriers from the deepest traps which capture the carriers at least once. However, usually the trap parameter $\omega(\epsilon)$ is assumed to be a strongly decreasing function of depth $\epsilon$, and then the transit temperature given by Eq. (28) corresponds to the highest maximum of the TSC spectrum. This is the case expected for dispersive-transport systems, for which the concentration of traps is considered to be exponentially decreasing with depth. If there is a trap which captures the carrier once (a critical trap), the maximum temperature corresponds to its release temperature.

We shall consider another situation where the distribution $n(\epsilon)$ has an empty region with no traps having a depth between $\epsilon_{1}$ and $\epsilon_{2}$. This is equivalent to a more generally accepted concept of two different processes or sets of traps, each of them distributed, but both well separated in energy. One can accept also that in the other set the distribution is characterized by different parameters. We further assume that $\epsilon_{c}>\epsilon_{i}$, in other words, the first set of traps with energies $0 \leqslant \epsilon \leqslant \epsilon_{1}$ does not contain the critical trap. When the temperature reaches $T_{r}\left(\epsilon_{1}\right)$, the release temperature of the trap with energy $\epsilon_{1}$ carriers from traps below $\epsilon_{1}$ will be liberated and trapped in traps deeper than $\epsilon_{2}$. Upon further temperature increase the current will fall because no full traps between $\epsilon_{1}$ and $\epsilon_{2}$ are available for liberation. We then obtain a nontransport peak with maximum temperature around $T_{r}\left(\epsilon_{1}\right)$. If the second set of traps with $\epsilon>\epsilon_{2}$ contains the critical trap, the second peak to appear will be a transport peak of the dispersive type, and if $\epsilon_{1}<\epsilon_{c}<\epsilon_{2}$, the second peak also will be a nontransport peak with maximum temperature around $T_{r}\left(\epsilon_{2}\right)$. The se considerations show that unlike the classical model ${ }^{3}$ the TSC spectrum does not reflect the shape of the trap distribution, but eventually monitors its edges if the set of traps under consideration does not contain the critical trap. One should also note that the two cases of nontransport peaks described above, in spite of different shapes, correspond to the classical TSC maxima with fast retrapping and no retrapping conditions, respectively.

\section{APPLICATIONS}

The theory will now be applied to particular cases of a single trapping level and of a continuum of traps exponentially distributed in energy. For simplicity the attempt-to-escape frequency will be assumed to be activation-energy independent, and thus the function $C(\epsilon)$ will have a constant value $C_{0}$.

\section{A. Single-level case}

In this case, for the distribution $n(\epsilon)$ we take a delta function

$$
n(\epsilon)=n_{t} \delta\left(\epsilon-\epsilon_{1}\right)
$$

which corresponds to single level of traps with depth $\epsilon_{1}$ and concentration $n_{t}$. Those traps will have the release temperature $T_{r}\left(\epsilon_{1}\right)$ defined as in Eq. (4),

$$
\frac{C_{0} N_{0}}{\beta} \int_{T_{0}}^{T_{r}\left(\epsilon_{1}\right)} \exp \left(-\epsilon_{1} / k T\right) d T=1 .
$$

Using Eq. (19) and (20) we obtain

$$
\left.\begin{array}{l}
v(T)=\mu E \\
\lambda(T)=C_{0} n_{t}=\tau_{R}^{-1}
\end{array}\right\} \text { for } T<T_{r},
$$

and

$$
\left.\begin{array}{l}
\left.v(T)=\mu E\left[1+\frac{n_{t}}{N_{0}} \exp \left(\frac{\epsilon_{1}}{k T}\right)\right]^{-1}\right\} \text { for } T \geqslant T_{r}, \\
\lambda(T)=0
\end{array}\right\}
$$

where $\tau_{R}$ is the free lifetime of a carrier in the transport states.

Separating the integral in Eq. (28) into two integrals with intermediate limit $T_{r}$ we obtain the maximum condition

$$
C \mu E \tau_{R}+\frac{\mu E}{\beta} \int_{T_{r}}^{T_{m}}\left[1+\frac{N_{t}}{N_{0}} \exp \left(\frac{\epsilon_{1}}{k T}\right)\right]^{-1} d T=L,
$$

where

$$
C=1-\exp \left[-\left(T_{r}-T_{0}\right) / \beta \tau_{R}\right] \simeq 1,
$$

since $\tau_{R}$ is much smaller than the time $\left(T_{r}-T_{0}\right) / \beta$ necessary to reach the release temperature. The term $\mu E \tau_{R}$ represents the distance traveled by a carrier, since it is generated at the surface and before it is trapped. The second term is the distance traveled by the carrier in equilibrium between the traps and transport states until it reaches the counter electrode, which corresponds, by definition, to the maximum temperature. In this case the velocity of the mean is equal to the velocity of the average carrier, and we deal with a nondispersive peak. Neglecting the small initial distance before the first trapping event $\mu E \tau_{R}$ and considering trap-controlled transport, the maxi- 

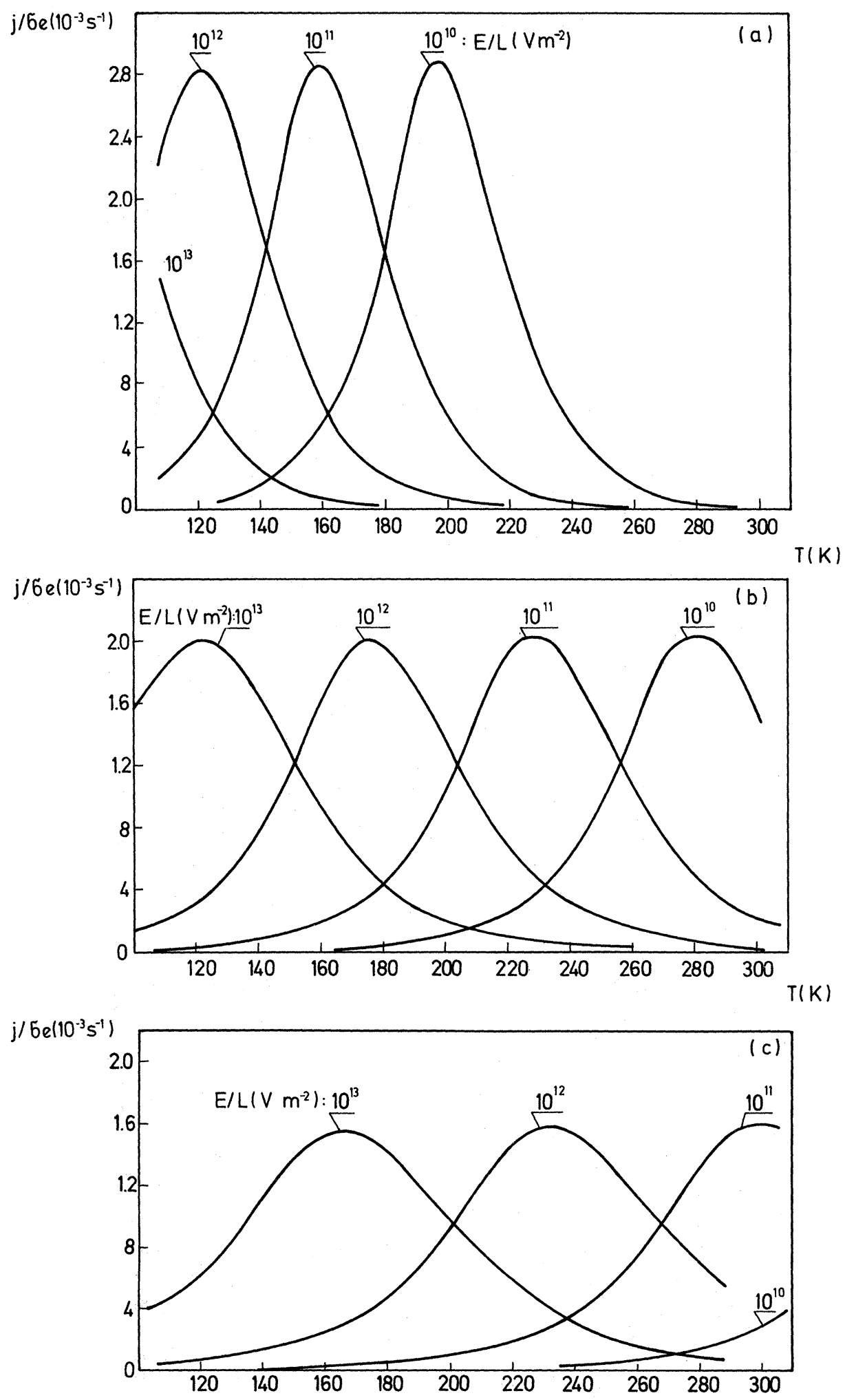

FIG. 2. The transport TSC peaks shifting with field and thickness plotted for three values of characteristic depths: (a) $\epsilon_{0}=0.05 \mathrm{eV}$, (b) $\epsilon_{0}=0.07 \mathrm{eV}$, and (c) $\epsilon_{0}=0.09 \mathrm{eV}$. Parameters of the maxima: $\mu=10^{-6} \mathrm{~m}^{2} / \mathrm{V} \mathrm{s}, N_{0}=10^{25} \mathrm{~m}^{-3}, C_{0}$ $=5 \times 10^{-12} \mathrm{~m}^{3} / \mathrm{s}, \beta=0.15 \mathrm{~K} \mathrm{~s}^{-1}$. 
mum condition becomes

$$
T_{m}=\epsilon_{1} / k \ln \left\{\frac{C_{0} N_{0} k T_{m}^{2}}{(1+R) \beta \epsilon_{1}}\right\},
$$

where the retrapping parameter $R=C_{0} n_{t} L / \mu E$ represents the number of consecutive trappings and detrappings of a carrier until it reaches the collecting electrode. As was expected, in the case of a single level we arrive at a known formula, which has been derived, ${ }^{6}$ however, based on dif-. ferent formalism.

Anticipating further discussion one should note an interesting property of this maximum condition; for $R \gg 1 \mathrm{Eq}$. (42) can be rewritten as

$$
T_{m}^{-1}=C+k / \epsilon_{1} \ln (E / L),
$$

which implies that the maximum temperature of the TSC transport peak should not only depend on the electric field, which has been observed experimentally, ${ }^{12,13}$ but also on the thickness of the sample. Although for a single level the dependence is rather weak, it cannot be at all neglected for the dispersive case (see Fig. 4), which has a strong impact on the analysis of experimental results. It is also interesting to note that the same maximum condition can be obtained assuming that $T_{m}$ is reached when a carrier traveling with temperaturedependent drift velocity $\mu_{d}(T) E$ moves through a distance equal to the sample thickness:

$$
\frac{1}{\beta} \int_{T_{r}}^{T_{m}} \mu_{d}(T) E d T=L,
$$

where $\mu_{d}(T)$ is the trap-controlled drift mobility in a nondispersive transport ${ }^{14}$

$$
\mu_{d}=\mu \Theta, \quad \Theta=1 /\left[1+\frac{n_{t}}{N_{0}} \exp \left(\frac{\epsilon_{1}}{k T}\right)\right] .
$$

This result clearly points out the direct analogy between the time-of-flight and transport TSC. Remembering that in TSC the temperature is not an independent variable, but a function of time, it can be seen that the release temperature in TSC corresponds to lifetime in an isothermal experiment, and the transit-temperature maximum is the analog of transit time. With the replacement of these variables, Eq. (44) can be converted into the isothermal equation of transport.

One should note that the lower limit of integration in Eqs. (41) and (44) is the release temperature $T_{r}$ of the trap $\epsilon_{1}$. This means that these equations describe the shift of the maximum temperature of the TSC peak, due to delayed transport through the distance $L$. In the case of fast retrapping, $L$ can be assumed to be zero, and thus $T_{m}=T_{r}$, as to be expected.

\section{B. Exponential trap distribution}

It was shown ${ }^{8}$ that a dispersive trace in the timeof-flight signal is obtained, assuming the exponential distribution of traps,

$$
n(\epsilon)=n_{0} \exp -\left(\epsilon / \epsilon_{0}\right),
$$

where $\epsilon_{0}$ denotes the characteristic depth of the distribution. This distribution fulfills the relation $\omega(\epsilon) \sim r(\epsilon)^{\alpha}$, the condition necessary to obtain universality of the current shape. ${ }^{8}$ It can be shown using Eqs. (8) and (9) that

$$
\exp \left(-\epsilon / \epsilon_{0}\right)=[\exp (-\epsilon / k T)]^{k T / \epsilon_{0}},
$$

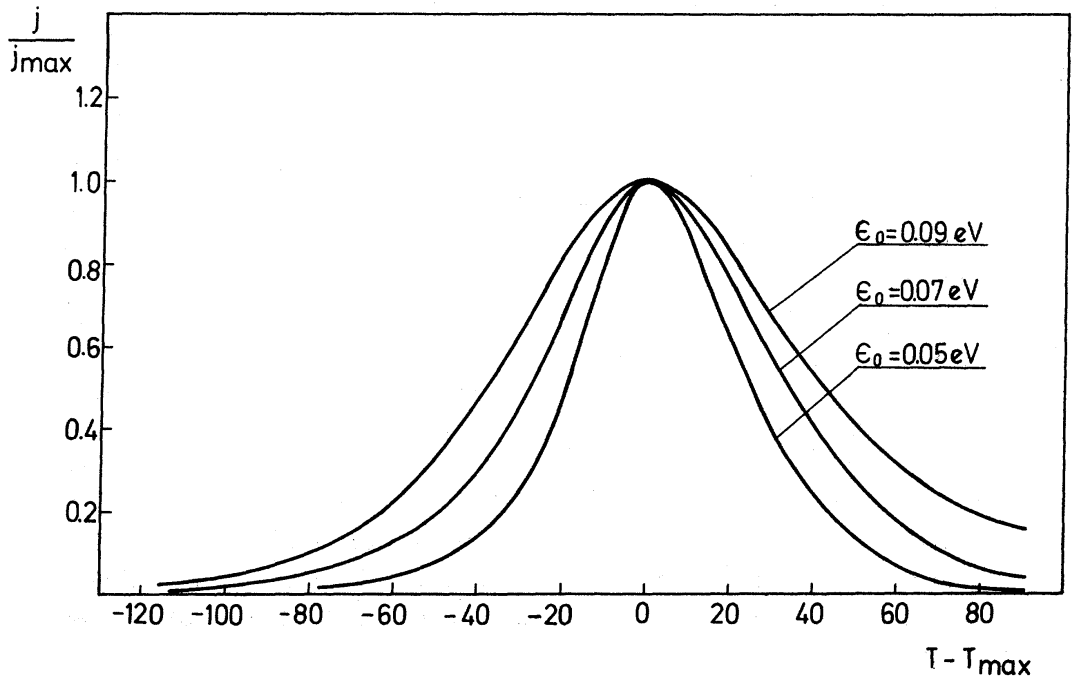

FIG. 3. Effect of the parameter of distribution $\epsilon_{0}$ on the shape of transport TSC maxima. Plots are normalized to the maximum value and shifted along the abscissa. Parameters are the same as in Fig. $2, E / L=10^{12} \mathrm{~V} \mathrm{~m}^{-2}$. 
and that the dispersion parameter $\alpha$ turns out to be equal to $k T / \epsilon_{0}$. The TSC transport peaks calculated by means of Eqs. (30) and (32) for different values of the parameter $\epsilon_{0}$ are depicted in Figs. 2 and 3.

Applying the exponential distribution to the maximum condition derived previously (36), we obtain an equation for the maximum temperature of dispersive transport peak:

$$
T_{m}=\bar{\epsilon}\left(T_{m}\right) / k \ln \left(\frac{C_{0} N_{0} k T_{m}^{2}}{\beta \bar{\epsilon}\left(T_{m}\right)}\right),
$$

where the critical depth is

$$
\epsilon_{c}=\bar{\epsilon}\left(T_{m}\right)=\epsilon_{0} \ln \left(C_{0} n_{0} \epsilon_{0} / \mu\right)-\epsilon_{0} \ln (E / L) .
$$

Equation (48) can be presented with good accuracy in a form

$$
T_{m}=C_{1}-C_{2} \ln (E / L)
$$

and

$$
C_{1} / C_{2}=\ln (1 / \tau \mu),
$$

where $\tau^{-1}=C_{0} n_{0} \epsilon_{0}$ 。

This implies that the maximum temperature of the transport TSC peak is thickness and field dependent, and in the case of dispersive transport, which is very likely found in amorphous materials, $T_{m}$ is proportional to $\ln (E / L)$, as can be seen in Fig. 4. This is a rather important conclusion because it implies that the position of the transport peak depends not only on experimental conditions, such as heating rate or electric field, but also on the thickness of the sample, which has been neglected in the analysis of TSC results. It might have been a source of confusion in comparing results for different samples. This result should be expected for the transport peak, since the maximum temperature is an analog of transit time in isothermal experiments, and transit time obviously depends on thickness. In the case of dispersive transport this dependence is superlinear due to the thickness dependence of the critical trap depth. Because the thickness dependence of $T_{m}$ in Eq. (48) results also from the dependence of $\epsilon_{c}$ on thickness, the superlinearity of the transit time and thickness dependence of $T_{m}$ are correlated in the sense that they are caused by the same effect.

For a nondispersive transport peak the maximum temperature also depends on thickness, but the activation energy around the maximum will be thickness independent in this case. This is especially important when one attempts to ascribe an activation energy obtained by partial heating around the maximum to a particular trapping level. The thickness dependence of $T_{m}$ for the dispersive-transport case has been shown by numeri-

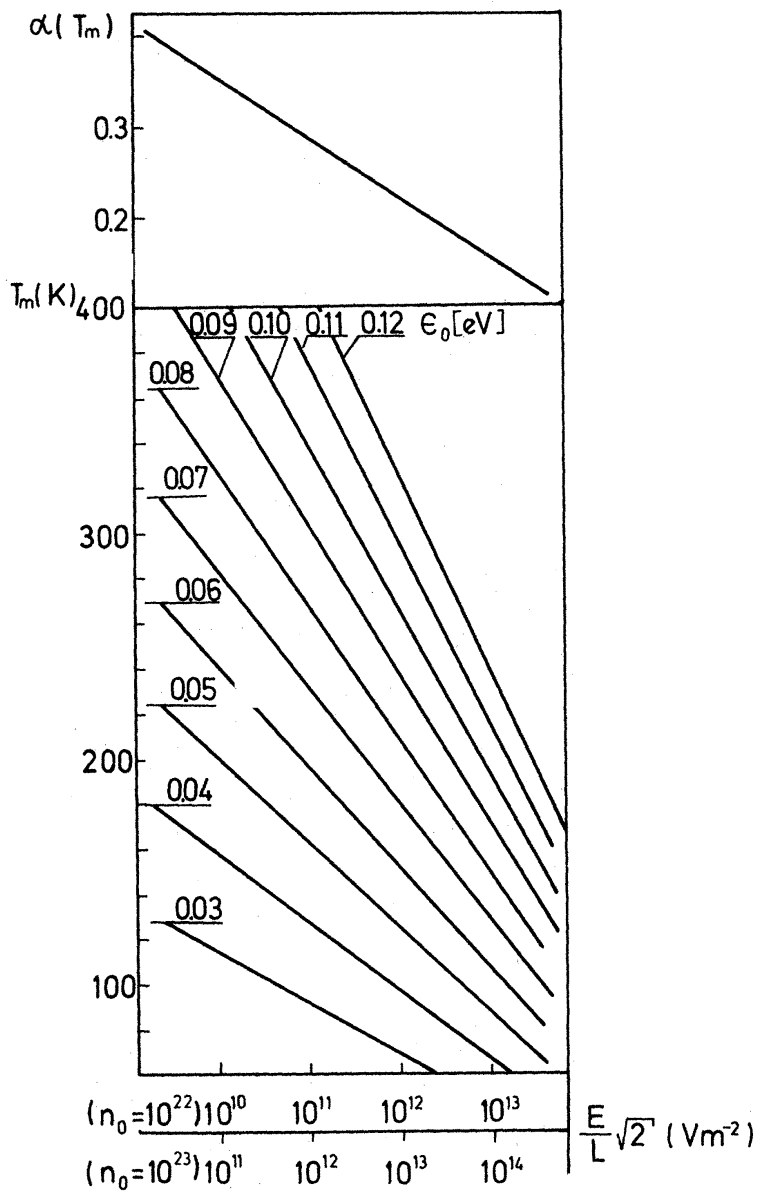

FIG. 4. Variation of the maximum temperature $T_{m}$ of a dispersive TSC peak with field and thickness and the corresponding plot of the dispersion parameter $\alpha\left(T_{m}\right)$. Parameters used are: $N_{0}=10^{25} \mathrm{~m}^{-3}, C_{0}=5 \times 10^{-12} \mathrm{~m}^{3} \mathrm{~s}^{-1}$, $\mu=10^{-6} \mathrm{~m}^{2} / \mathrm{V} \mathrm{s}, \beta=0.15 \mathrm{~K} \mathrm{~s}^{-1}$. The effect of trap density $n_{0}$ is illustrated by shifting the abscissa. Note that the dispersion parameter $\alpha$ at $T_{m}$ depends on field and thickness and is practically independent on the characteristic depth $\epsilon_{0}$.

cal calculations, ${ }^{11}$ and the present theory yields the analytical expression for this dependence, allowing us to derive the material parameters from the experimental results.

In order that those calculations be valid for a particular experimental TSC peak, it must be first proved that we deal with a real transport peak. The thickness and field dependences of $T_{m}$ are reliable tests for this hypothesis. Figure 4 shows that for typical values of the material parameters such as $\mu=10^{-6} \mathrm{~m}^{2} / \mathrm{V} \mathrm{s}, N_{0}=10^{15} \mathrm{~m}^{-3}$, and $C_{0}=5 \times 10^{-12} \mathrm{~m}^{3} / \mathrm{s}$, this dependence is rather strong in the dispersive case. Since traps which are charged when empty ${ }^{15}$ are able to modify the field dependence of $T_{m}$, in this case it is better to keep the field constant and vary the thickness. If 
Poole-Frenkel or polaron-type field dependence is found, the plots of $T_{m}$ against $\ln L$ will be of different slopes for each field.

The next step in analysis is to find whether we deal with the dispersive or single-level transport peak (the latter is, however, rather unlikely in disordered systems). For single level the maximum condition is

$$
T_{m}^{-1}=C+\left(k / \epsilon_{1}\right) \ln (E / L) \text { for } R \gg 1,
$$

where

$$
C=\frac{k}{\epsilon_{1}} \ln \left(\frac{N_{0} k T_{m}^{2} \mu}{\beta n_{t} \epsilon_{1}}\right) .
$$

If $C$ is not much larger than the second term in Eq. (52), for a limited range of thickness $L$, one can obtain experimentally the linear dependence in a plot of $T_{m}$ vs $\ln (E / L)$, which is characteristic of the dispersive peak. Hence, linear dependence of $T_{m}$ on $\ln (E / L)$ can be considered a general property of the transport peaks, at least in a limited range of thickness. The thickness dependence of $T_{m}$ in the case of a dispersive peak is, however, stronger. The experimental slope $s$ of a plot of $T_{m}^{-1}$ vs $\ln (E / L)$ must, for a single level, fulfill the relation

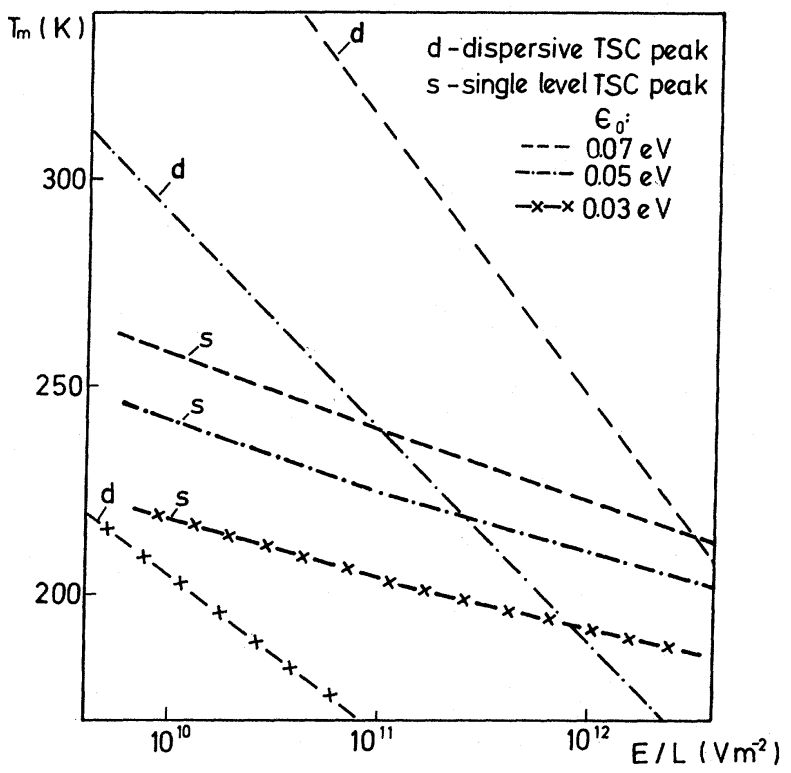

FIG. 5. Variation of maximum temperature $T_{m}$ of a transport TSC peak with field and thickness for dispersive and single-level cases calculated assuming $\mu$ $=10^{-6} \mathrm{~m}^{2} \mathrm{~V}^{-1} \mathrm{~s}^{-1}, N_{0}=10^{25} \mathrm{~m}^{-3}, C_{0}=5 \times 10^{-12} \mathrm{~m}^{3} \mathrm{~s}^{-1}, \beta$ $=0.15 \mathrm{~K} \mathrm{~s}^{-1}$. For dispersive transport $n_{0}=10^{22} \mathrm{~m}^{-3}$, and the characteristic depth $\epsilon_{0}$ is varied, while for single level $\epsilon_{1}=0.6 \mathrm{eV}$, and the trap density is adjusted to correspond to the dispersive transport in each case $n_{t}$ $=n_{0} \exp \left(-\epsilon_{1} / \epsilon_{0}\right)$.

$$
s \leqslant k / \epsilon_{\mathbf{I}} \text {. }
$$

Thus if the experimental value of $\bar{\epsilon}\left(T_{m}\right)$, provided by partial heating [see Eq. (62)], is higher than $k / s$, we certainly deal with a dispersive transport peak. The comparison of thickness dependence of $T_{m}$ for the two kinds of transport TSC peaks is presented in Fig. 5 .

Having performed the partial heating experiment around $T_{m}$ we can calculate the characteristic depth $\epsilon_{0}$, transforming Eq. (49),

$$
\epsilon_{0}=\bar{\epsilon}\left(T_{m}\right) / \ln \left(\frac{L}{\tau \mu E}\right) \text {, }
$$

where $\ln (1 / \tau \mu)$ can be found from the slope-tointercept ratio of $T_{m}$ vs $\ln (E / L)$ plot [Eq. (51)]. The characteristic depth allows us to calculate the dispersion parameter $\alpha=k T / \epsilon_{0}$. An independent way of calculating $\epsilon_{0}$ from initial rise and partial heating is presented in the next chapter.

Finally, since the maximum temperature is also the release temperature of a critical trap, as was shown in Sec. III C, we have

$$
T_{m}=\bar{\epsilon}\left(T_{m}\right) / k \ln \left(\frac{C_{0} N_{0} k T_{m}^{2}}{\beta \bar{\epsilon}\left(T_{m}\right)}\right),
$$

which allows us to obtain $C_{0} N_{0}$. Provided with experimental values for $\left(C_{0} N_{0}\right),(\mu \tau)^{-1}$, and $\epsilon_{0}$, we can obtain the transit time (corresponding to isothermal time-of-flight experiments) at each temperature:

$$
t_{\tau} \simeq \frac{1}{C_{0} N_{0}}\left(\frac{L}{\mu \tau E}\right)^{c_{0} / R T}
$$

and thus evaluate the drift mobility.

$$
\text { C. Partial-heating and initial-rise techniques }
$$

Evaluation of activation energies associated with the maxima has always been an important part in the analysis of TSC spectra. In particular the initial-rise and partial-heating techniques are widely used for this purpose. In classical nontransport models the slope of the logarithm of initial current, plotted against $1 / k T$ for temperatures sufficiently far below the maximum, yields in the case of a single trap its activation energy. For the distribution of traps the slope is lower, usually reduced by a factor correlated with the distribution width. ${ }^{16}$ In this case the partialheating technique has been found useful since it yields the true activation-energy value, varying, however, with temperature.

The partial-heating technique consists in interrupting the TSC run at some temperature $T_{c}$, cooling the sample, and making a new run until $T_{c}+\Delta T$ is reached, but without repeating the generation step at $T_{0}$. In the case of the TSC trans- 
port peak, when interrupting the run at $T_{c}$ traps having a depth less than the release energy, $\bar{\epsilon}\left(T_{c}\right)$ are already in quasistationary state $(\partial \rho / \partial T \simeq 0)$. Because the release temperatures are lower than $T_{c}$ for those traps, they are practically exhausted, since the lifetime $\tau_{R}$ before being immobilized in deep traps, defined as

$$
\tau_{R}^{-1}\left(T_{c}\right)=\int_{\tilde{\epsilon}\left(T_{c}\right)}^{\infty} C(\epsilon) n(\epsilon) d \epsilon,
$$

is much shorter than the time scale of the experiment. In the following run of partial heating the main contribution to the current has the carriers released from traps with the release temperatures between $T_{c}$ and $T_{c}+\Delta T$. Using the average concentrations we can write

$$
j(T) \sim p_{0}(T),
$$

and

$$
\beta \frac{d p_{0}(T)}{d T}=\frac{\rho(T, \bar{\epsilon})}{\bar{\tau}}-\frac{p_{0}(T)}{\tau_{R}\left(T_{c}\right)},
$$

where

$$
\bar{\tau}^{-1}=C(\epsilon) N_{0} \exp \left[-\bar{\epsilon}\left(T_{c}\right) / k T\right] .
$$

Assuming that $\tau_{R}$ is small we get, solving Eq. (60),

$$
j(T) \frac{1}{\tau} \exp \left(-\frac{1}{\beta} \int_{T_{c}}^{T} \frac{1}{\bar{\tau}} d T\right),
$$

and around $T_{c}$ we can neglect the exponential factor, obtaining

$$
\ln j(T) \sim-\bar{\epsilon}\left(T_{c}\right) / k T .
$$

This last relation implies that the partial-heating experiment interrupted at $T_{c}$ yields approximately the release energy $\bar{\epsilon}\left(T_{c}\right)$ at this temperature. One could expect that the thermal equilibrium between transport states and shallow trapping levels could affect the experimentally obtained activation energy. This influence is, however, small and can be completely neglected for temperatures below $\epsilon_{0} / 2 k$. From the definition of release energy (4) we have

$$
\bar{\epsilon}(T)=k T \ln \left(\frac{\nu(\bar{\epsilon}) k T^{2}}{\beta \bar{\epsilon}(T)}\right),
$$

and thus $\bar{\epsilon}\left(T_{c}\right)$ is an increasing function of $T_{c}$. Since the logarithmic term varies weakly with temperature, $\bar{\epsilon}\left(T_{c}\right)$ is proportional to $T_{c}$ with very good accuracy (see Fig. 5).

It is interesting to consider the behavior of the partial-heating plots in the case of the interrupted distribution having an empty region for $\epsilon_{1}<\epsilon<\epsilon_{2}$, as discussed in Sec. II C. The partial-heating curves yield the $\bar{\epsilon}\left(T_{c}\right)$ upon increasing $T_{c}$, until it reaches $T_{r}\left(\epsilon_{1}\right)$. In further runs, only a narrow range of traps with activation energies around $\epsilon_{1}$ are still being emptied from the rest of carriers, which are then deep-trapped in traps with $\epsilon \geqslant \epsilon_{2}$. The partial-heating currents will have decreasing amplitude, and their slopes will yield $\epsilon_{1}$ for several consecutive runs, unless $T_{c}$ is sufficiently close to $T_{r}\left(\epsilon_{2}\right)$. The plot of experimentally obtained activation energy versus $T_{c}$ will then consist of a part increasing with temperature, followed by a plateau, and eventually the next increasing part. Such a property of the partialheating results, which are often observed in experiments, ${ }^{17}$ attains a new interpretation based on the concepts of the transport TSC peak.

The preceding conclusions are valid for a general distribution, provided that the trap parameter of capture $\omega(\epsilon)$ is a decreasing function of

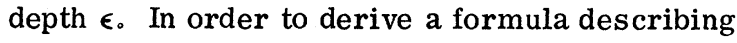
the initial rise of current we shall turn back to the exponential distribution of traps. Using Eq. (31) for the motion of the mean $\bar{x}$, the solution of which is given by Eqs. (48) and (49), putting $\bar{x}(T)$ for $L$ and $T$ for $T_{m}$, one obtains

$$
j \sim \beta \frac{d \bar{x}}{d T} \sim C \exp \left(\frac{\bar{\epsilon}(T)}{\epsilon_{0}}\right) .
$$

By plotting $\ln j$ vs $1 / T$ one can derive the activation energy. Denoting the experimentally obtained activation energy as $A_{I}(T)$ we can write

$$
-\frac{d(\ln j)}{d(1 / k T)}=A_{I}(T)=\bar{\epsilon}(T) \alpha(T),
$$

where $\alpha(T)$ is the dispersion parameter equal to $k T / \epsilon_{0}$. This shows that $A_{I}(T)$ is approximately proportional to $T^{2}$. Denoting $A_{p}(T)$, the partialheating activation energy, we can rewrite $\mathrm{Eq}$. (65) as

$$
A_{I}(T)=A_{P}(T) \alpha(T) .
$$

It can be seen from the above that the initial

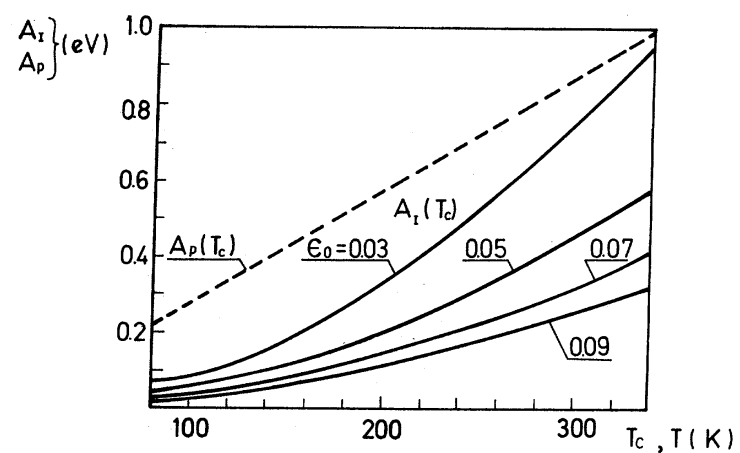

FIG. 6. Comparison of activation energies obtained from initial rise $\left(A_{I}\right)$ and by partial-heating technique $\left(A_{p}\right)$ with characteristic depth of a distribution $\epsilon_{0}$ as a parameter for $\nu=10^{13} \mathrm{~Hz}$ and $\beta=0.15 \mathrm{~K} \mathrm{~s}^{-1}$. 
rise current, when plotted in Arrhenius coordinates, does not yield a straight line; the activation energy can, however, be found graphically at any temperature from the slope of the line, or by numerical derivation. Having the values of $A_{p}(T)$ obtained from partial heating one can find the dispersion parameter $\alpha$, and thus the characteristic depth of the distribution, from the plot $A_{I}(T) / A_{P}(T)$ vs $T$. This method yields an independent estimate of the characteristic depth $\epsilon_{0}$, which can be used in Eqs. (55)-(57). Figure 6 presents the variation of "initial-rise" and "partial-heating" activation energies with temperature for several values of the parameter $\epsilon_{0}$.

\section{SUMMARY AND CONCLUSIONS}

A set of equations describing the properties of the TSC transport peak is derived, based on the theory of transient photoconductivity and with the use of the concepts of release energy and critical trap. The transport TSC peak is a peak which is controlled by the transport of charge carriers through the sample, stopped by multiple trapping and detrapping events, and its position on the temperature scale is field and thickness dependent. The temperature-dependent release energy is the one which divides the continuum of traps into a shallow-trap region, where carriers are in equilibrium with transport states, and the deeptrap region, where they are quasipermanently trapped. The critical trap is a trap which captures a carrier approximately once in its travel through the sample, i.e., a trap for which the mean time between release and subsequent retrappings is equal to the time which a carrier needs to travel a distance of the sample thickness.

The basic concepts behind the theory of a TSC transport peak are very similar to that used in the theory of transient photoconductivity. In order to facilitate the analysis of properties of the transport peak, concepts of release temperature and transit temperature are introduced. The release temperature $T_{r}$, an equivalent of the lifetime in an isothermal experiment, is a temperature, depending on initial temperature $T_{0}$, heating rate $\beta$ and trap depth $\epsilon$, at which the release rate from this trap reaches its maximum.

In fact, release energy and release temperature are symmetrical concepts, in the sense that for any given temperature one can find in a continuum of traps the one having the release energy at this temperature, and for any particular trap its release temperature can be defined. The transit temperature, an equivalent of transit time in timeof-flight experiments, is a temperature at which the maximum of TSC transport peak is obtained.

The general theory is applied to the dispersive transport case, which is characterized by the presence of a critical trap in a sequence of traps, from which carriers are released during experiment. This type of transport exhibits the property of universality, for which a necessary condition is that the capture probability is a strongly decreasing function of a release probability.

The principal conclusions from this study are:

(i) The critical-trap criterion (CTC), defined for isothermal transport, holds also for a dispersive transport TSC peak: Such a peak implies the existence of a trap, bracketed by the distribution of traps, which captures the carrier once. The release temperature of this trap is the maximum temperature (transit temperature) of the peak.

(ii) The release energy at maximum temperature is equal to the depth of a critical trap, which means that $T_{m}$ is reached when carriers released from traps having their release temperature at $T_{m}$ travel through the sample without being retrapped in traps with depth $\bar{\epsilon}\left(T_{m}\right)$ or deeper.

(iii) The maximum temperature of a transport peak depends on field strength and thickness of the sample; for the single level this dependence is linear in a $T_{m}^{-1}$ vs $\ln (E / L)$ plot (for $R \gg 1$ ), while for an exponential distribution of traps the dependence is stronger, and $T_{m}$ is proportional to $\ln (E / L)$.

(iv) The shape of the transport peak below maximum can be considered as a superposition of single-release peaks, the carriers released from each trap being trapped in the deeper ones. The rise of current in a partial-heating experiment can be described with a good approximation considering the kinetics of carrier release from a single trap $\left(T_{c}\right)$ and fast retrapping. The slope of the partial heating current yields in an Arrhenius plot an activation energy $A_{p}\left(T_{c}\right)$ equal to the release energy $\bar{\epsilon}\left(T_{c}\right)$, where $T_{c}$ is a temperature at which heating is interrupted. The activation energy $A_{p}\left(T_{c}\right)$ is approximately linear with $T_{c}$. The current shape above maximum temperature $T_{m}$ can be considered as the superposition of single release peaks with no retrapping condition.

(v) The partial-heating activation energy at $T_{m}$, $A_{p}\left(T_{m}\right)$, is equal to critical trap depth $\epsilon_{c}$ and as such depends on field and thickness. The slope of a straight line of $A_{p}\left(T_{m}\right)$ against $\ln (E / L)$ yields the characteristic depth of the exponential trap distribution $\epsilon_{0}$. The activation energy $A_{p}\left(T_{m}\right)$ is the activation energy of the drift mobility and the distribution parameter $\epsilon_{0}$ yields the dispersion parameter $\alpha\left(T_{m}\right)=k T_{m} / \epsilon_{0}$ of the time-of-flight signal at $T_{m}$.

(vi) The initial rise of current does not give a straight line in Arrhenius coordinates; activation energy $A_{I}(T)=d \ln j / d(1 / k T)$ derived from this plot 
is lower than $\bar{\epsilon}(T) ; A_{I}(T)=\bar{\epsilon}(T) \alpha(T)$, where $\alpha(T)$ is the parameter of dispersion in time-of-flight experiments. This observation allows us to find the dispersion parameter experimentally from the TSC, comparing the initial-rise and partialheating activation energies.

(vii) When the continuous trap distribution is interrupted, having a region with no traps $\epsilon_{1}<\epsilon$ $<\epsilon_{2}$, which corresponds to two distributed sets of traps separated in energy, each region produces a TSC maximum. The peak which corresponds to a region not containing the critical trap is a nontransport peak. The plot of partial-heating activation energies vs $T_{c}$ will have a flat portion when the release temperature of trap $\epsilon_{1}$ is reached。

(viii) The theory of the transport peak developed for a continuous distribution is applicable also for a discrete set of traps. If the trap parameter $\omega(\epsilon)$ is a strongly decreasing function of depth $\epsilon$, as for exponential distribution of traps', the transit temperature is the maximum temperature of the highest peak.
* Permanent address: Instituto de Estructura de la Materia, Consejo Superior de Investigaciones Cientificas Serrano 119, Madrid, Spain.

${ }^{1}$ T. A. T. Cowell and J. Woods, Br. J. Appl. Phys. 18, 1045 (1967).

${ }^{2}$ R. R. Haering and E. N. Adams, Phys. Rev. 117, 451 (1960).

${ }^{3}$ J. G. Simmons, G. W. Taylor, and M. C. Tam, Phys. Rev. B 7, 3714 (1973).

${ }^{4}$ I. Chen, J. Appl. Phys. 47, 2988 (1976).

${ }^{5}$ A. Samoć, M. Samoć, and J. Sworakowski, Phys. Status Solidi A 36, 735 (1976).

${ }^{6}$ M. Samoć and A. Samoć, Phys. Status Solidi A $\underline{57}, 667$ (1980).

${ }^{7}$ H. Scher and E. W. Montroll, Phys. Rev. B 12,2455 (1975).

${ }^{8}$ F. W. Schmidlin, Phys. Rev. B 16, 2632 (1977).

${ }^{9}$ J. Noolandi, Phys. Rev. B 16, 4474 (1977).
${ }^{10}$ V. I. Arkhipov, M. S. Iovu, A. I. Rudenko, and S. D. Shutov, Phys. Status Solidi A 54, 67 (1979).

${ }^{11} \mathrm{~J}$. K. Jeszka, M. Zieliński, and M. Kryszewski, in Charge Storage, Charge Transport and Electrostatics With Their Applications, edited by Y. Wada, M. M. Perlman, and H. Kokado (Elsevier, Amsterdam and Kodansha, Tokyo, 1979), p. 287.

${ }^{12}$ A. P. Kulshreshta and I. J. Saunders, J. Phys. D $\underline{8}$, 1787 (1975).

${ }^{13}$ A. Samoć and J. Samoć, J. Electrostat. $\underline{8}, 121$ (1979).

${ }^{14}$ N. F. Mott and E. A. Davies, Electronic Processes in Non-Crystalline Materials (Clarendon, Oxford, 1971). ${ }^{15}$ M. Zieliński and M. Samoć, J. Phys. D 10, L105 (1977).

${ }^{16} \mathrm{H}$. Gobrecht and D. Hofman, J. Phys. Chem. Solids 27, 509 (1966).

${ }^{17}$ R. A. Creswell and M. M. Perlman, J. Appl. Phys. $\underline{41}$, 2365 (1970). 